\title{
OPEN Identification of a solo acylhomoserine lactone synthase from the myxobacterium Archangium gephyra
}

\author{
Hanan Albataineh, Maya Duke, Sandeep K. Misra, Joshua S. Sharp \& D. Cole Stevens ${ }^{\bowtie}$
}

Considered a key taxon in soil and marine microbial communities, myxobacteria exist as coordinated swarms that utilize a combination of lytic enzymes and specialized metabolites to facilitate predation of microbes. This capacity to produce specialized metabolites and the associated abundance of biosynthetic pathways contained within their genomes have motivated continued drug discovery efforts from myxobacteria. Of all myxobacterial biosynthetic gene clusters deposited in the antiSMASH database, only one putative acylhomoserine lactone (AHL) synthase, agpl, was observed, in genome data from Archangium gephyra. Without an AHL receptor also apparent in the genome of A. gephyra, we sought to determine if Agpl was an uncommon example of an orphaned AHL synthase. Herein we report the bioinformatic assessment of Agpl and discovery of a second AHL synthase from Vitiosangium sp. During axenic cultivation conditions, no detectible AHL metabolites were observed in A. gephyra extracts. However, heterologous expression of each synthase in Escherichia coli provided detectible quantities of $3 \mathrm{AHL}$ signals including 2 known AHLs, C8-AHL and C9-AHL. These results suggest that $A$. gephyra $A H L$ production is dormant during axenic cultivation. The functional, orphaned AHL synthase, Agpl, is unique to $A$. gephyra, and its utility to the predatory myxobacterium remains unknown.

Ubiquitous throughout soils and marine sediments, myxobacteria utilize cooperative features to facilitate uniquely social lifestyles and exhibit organized predation of microbial prey ${ }^{1-3}$. Often attributed to their predatory capabilities, an extraordinary number of biologically active specialized metabolites have been discovered from myxobacteria $^{4-8}$. Interest in this chemical space and the therapeutic potential associated with each elucidated natural product has motivated significant efforts towards continued discovery. Our recent survey of the unexplored, biosynthetic gene clusters from myxobacteria included in the antiSMASH database determined that the potential for such discovery from cultivable myxobacteria remains high ${ }^{9-12}$. An oddity reported by this survey was the presence of a solo acylhomoserine lactone (AHL) synthase within the genome of the myxobacterium Archangium gephyra ${ }^{12-14}$. As obligate cooperators numerous signaling systems have been associated with the coordination of myxobacterial motility and predation including A-signal, a quorum-like signal. However, no myxobacteria have been observed to produce AHL quorum signals.

Acylhomoserine lactone quorum signaling (QS) systems are abundant throughout Proteobacteria at-large ${ }^{15}$. Considered autoinducers, AHLs bind to LuxR-type receptors which in turn induce expression of LuxI-type AHL synthases. While a recent assessment of LuxR receptors included within or nearby specialized metabolite biosynthetic gene clusters (BGCs) reported the presence of a putative LuxR receptor from the marine myxobacterium Haliangium ochraceum DSM 14,365, no AHL quorum signals or functional LuxI-type AHL synthases have been reported from myxobacteria ${ }^{16}$. Additionally, the presence of putative LuxR receptors within numerous members of the genera Myxococcus and Corallococcus has been reported in a recent survey of myxobacterial signaling proteins ${ }^{17}$. Intriguingly, the model myxobacterium Myxococcus xanthus demonstrates enhanced predatory features when exposed to a variety of exogenous AHLs despite having no obvious LuxR receptor within its genome $^{18}$. This phenomenon, often referred to as "eavesdropping," has become a generally accepted cornerstone in hypotheses surrounding interspecies cross talk within polymicrobial communities, and the presence of solo or orphan LuxR receptors from species that do not produce AHL signals supports such communication ${ }^{18-25}$. Putative solo-LuxR transcription factors with no accompanying LuxI synthases account for the majority of annotated 
A

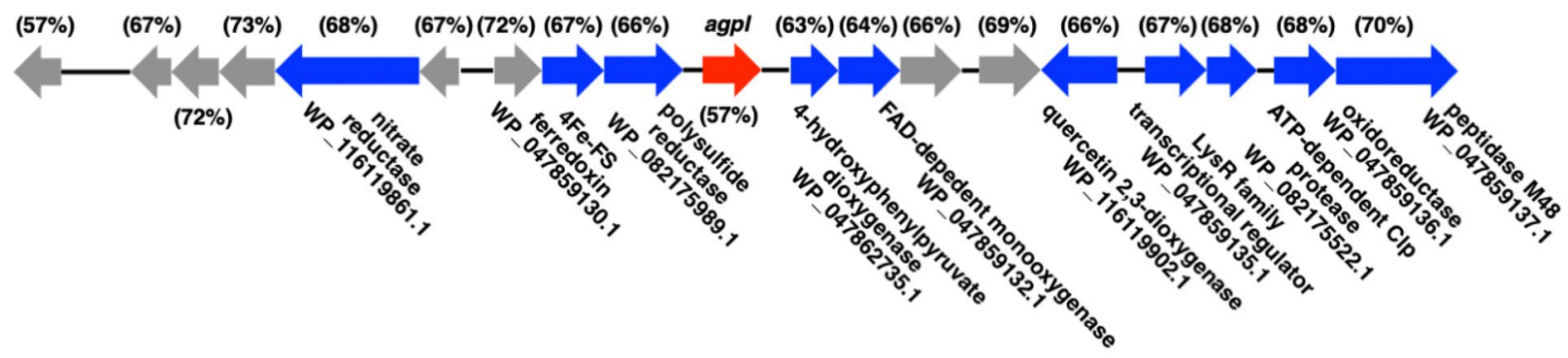

B

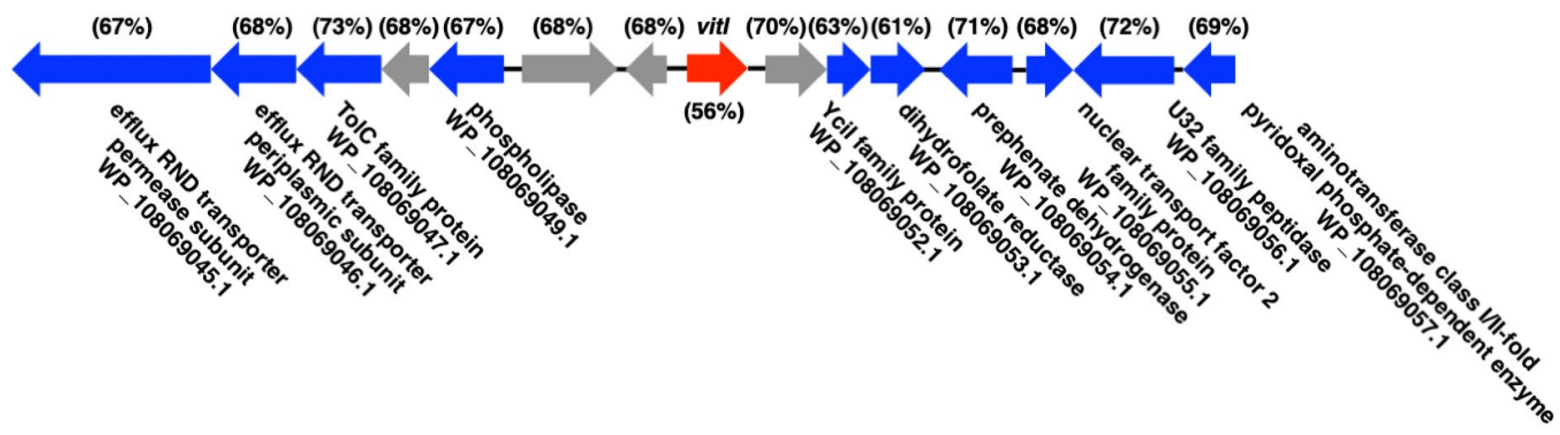

Figure 1. (A) Cluster 32 from A. gephyra deposited in the antiSMASH database which includes the putative AHL synthase, agpI. (B) Genomic context for vitI from Vitiosangium sp. All annotated features within NCBI are labelled and all hypothetical features are in grey. Percentage GC content for each gene within the cluster provided for comparison and depicted in parentheses.

LuxR proteins. However, as with M. xanthus, there are no LuxR receptors apparent in the genome of A. gephyra. This suggests that the observed LuxI-type synthase, AgpI, from A. gephyra is a solo-LuxI synthase. Considering the abundance of AHL QS systems throughout Proteobacteria other than myxobacteria, the uniqueness of this AHL synthase from A. gephyra, and the generalist diet of predatory myxobacteria that includes large swaths of AHL signaling proteobacteria, supports the assumption that agpI might have been acquired horizontally ${ }^{3,26-28}$. Conversely, the benefit AHL production might provide a predatory myxobacterium remains non-obvious. Herein we report bioinformatic analysis, functional assessment, and heterologous expression of the myxobacterial AHL synthase AgpI.

\section{Results}

Agpl is highly homologous to functional AHL synthases. Located in the $20.6 \mathrm{~kb}$ BGC referenced as cluster 32 from A. gephyra (NZ_CP011509) in the antiSMASH database (5.1.1) the 210 aa gene product, AgpI (WP_047862734.1), is annotated as a putative autoinducer synthesis protein homologous to the GNAT family $\mathrm{N}$-acetyltransferase, LuxI class of AHL synthases (Fig. 1A) ${ }^{9,10,20}$. None of the other annotated features neighboring agpI are obviously associated with AHL quorum signaling systems. Assessment of AgpI (WP_047862734.1) with highly homologous LuxI synthases using blastp against the non-redundant protein sequences database provided 2 additional putative AHL synthases within the genome of another myxobacterium, Vitiosangium sp. GDMCC 1.1324. These included a GNAT family $N$-acetyltransferase deemed VitI (WP_108069305.1) with 68.12\% identity when comparing amino acid sequence data with AgpI (Fig. 1B) and an annotated autoinducer synthase protein (WP_158502406.1) with 69.52\% identity with AgpI amino acid sequence ${ }^{29}$. The absence of genome data for Vitiosangium sp. in version 4.2.1 of the antiSMASH database explains the omission of this putative AHL synthase from our previous survey of myxobacterial biosynthetic space ${ }^{12}$. The next highest scoring sequence from this analysis, a GNAT family $N$-acetyltransferase (WP_055459978.1) from Chelatococcus sambhunathii has $96 \%$ coverage and $56.44 \%$ identity with $\mathrm{AgpI}^{30,31}$. When restricting the blastp search to only provide results from myxobacteria (taxid: 29), only 1 other GNAT $N$-acetyltransferase (WP_169850287.1) from Corallococcus exiguus was found to have $>45 \%$ identity with AgpI albeit at 50\% coverage. Interestingly, an alignment of these 4 putative LuxI-type AHL synthases from myxobacteria revealed that only AgpI and VitI possessed all 8 of the highly conserved residues associated with the LasI autoinducer domain (COG3916) (Fig. 2B). Alignment and phylogenetic analysis of AgpI and VitI against an assortment of 17 LuxI-type synthases experimentally validated to produce AHL QS molecules, suggests common ancestry with the AHL synthases LuxI, LasI, and TraI from Aliivibrio fischeri, Pseudomonas aeruginosa, and Rhizobium radiobacter, respectively (Fig. 2A, C) ${ }^{32-43}$. Utilizing the genomic enzymology web tool EFI-EST developed by the Enzyme Function Initiative (EFI) to construct a sequence similarity network (SSN) that included 1001 LuxI-type AHL synthases (PF00765) as nodes clustered in different groups and 124,346 edges, both AgpI and VitI are included in the central cluster family that contains the vast majority of homologous LuxI-type AHL synthases (Supplemental Figure 1) ${ }^{44}$. 


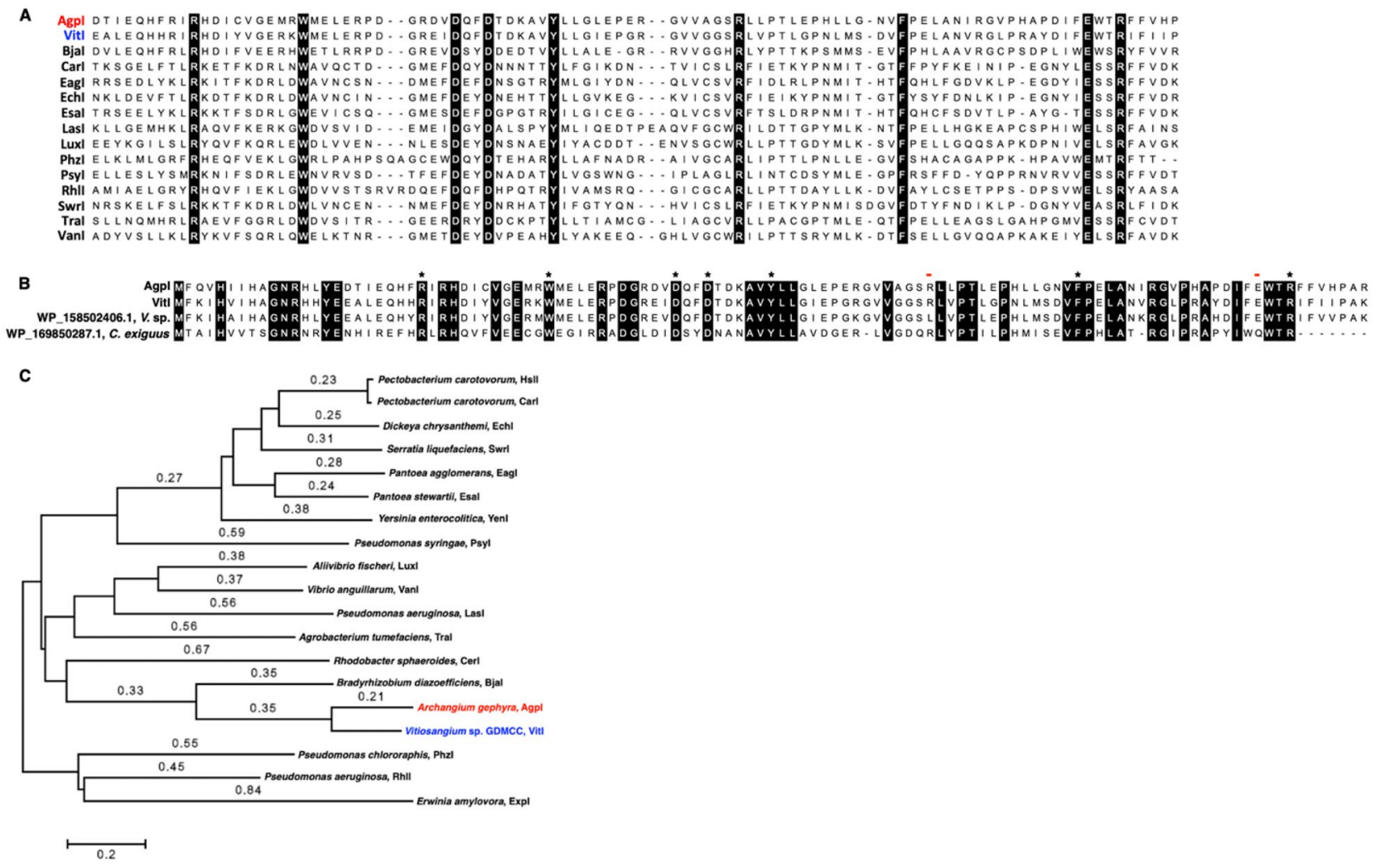

Figure 2. (A) Alignment of LuxI synthases including AgpI and VitI with conserved residues boxed in black. (B) Alignment of myxobacterial features homologous to AgpI with asterisks $\left(^{*}\right)$ indicating conserved residues associated with the LasI autoinducer domain (COG3916) and red lines (-) indicating residues from the LasI domain that are not conserved. (C) Minimum Evolution tree including AgpI and VitI rendered in MEGA7 using ClustalW aligned with AHL synthases experimentally confirmed to produce AHLs (68). Branch lengths $\leq 0.2$ not depicted.

Interestingly, although AgpI and VitI are highly homologous and both include all of the conserved residues associated with LuxI synthases, they share no neighboring similarities when comparing genomic context and surrounding features (Fig. 1). Considering the typical GC-rich genomes of myxobacteria, we sought to compare the GC content of the genes surrounding AgpI and VitI to determine if either were obviously less GC-rich which would support horizontal acquisition. The genomes of both A. gephyra and Vitiosangium sp are $69.4 \%$ and 68.3\% GC, respectively. The GC content of AgpI (57\%) and VitI (56\%) are indeed lower than the genome GC content of each myxobacterium as well as the averages of the genes surrounding them (Fig. 1). A comparison of the genomic context for AgpI (Supplemental Figure 2A) and VitI (Supplemental Figure 2B) as well as the LuxI/ GNAT $N$-acetyltransferases with the highest homology from our original blastp analysis of AgpI, including (WP_158502406.1) from Vitiosangium sp. (Supplemental Figure 2C), (WP_119746752.1) from Paracoccus sp., (WP_055459978.1) from Chelatococcus sambhunathii, and (WP_008839764.1) from Mesorhizobium alhagi (Supplemental Figure 2D), did not provide an obvious genomic island that might have been included in a hypothetical horizontal transfer event.

Utilizing the BiG-SCAPE platform ${ }^{45}$ which uses a modified iteration of clusterblast, we generated a similarity network from a total of 6627 biosynthetic gene clusters predicted to include LuxI-type AHL synthases from the antiSMASH database ${ }^{9,10}$ including annotated LuxI-type AHL synthases. The biosynthetic gene cluster from $A$. gephyra that includes AgpI appears as a singleton within the resulting similarity network and no pathway-level homology with the other 6627 AHL-containing gene clusters was observed (Supplemental Figure 3). Ultimately these analyses did not provide a clear candidate LuxI-containing gene cluster from which AgpI was acquired; these results do not preclude the possibility that AgpI was indeed acquired as a genetic insertion with various additional features including an associated LuxR-type receptor that have since been lost due to genome reduction. Overall, we suggest that the shared ancestry observed from phylogenetic analysis of AgpI and VitI with known LuxI synthases and highly conserved active site residues suggest both AgpI and VitI are indeed LuxI synthases as originally predicted by antiSMASH.

Absence of a cognate AHL receptor in the genome of $\boldsymbol{A}$. gephyra. While no obvious AHL-binding LuxR homolog was identified in the chromosome of A. gephyra, we sought to determine the presence of any potential AHL-binding domain using the conserved sequence for autoinducer binding domains (PF03472). Utilizing the blastp suite at NCBI, we assessed all 3014 domains within the Pfam database classified as autoinducer binding domains for homology against the deposited genome of $A$. gephyra ${ }^{14,46}$. No features within the proteome 
of A. gephyra were sufficiently homologous to be considered to include an autoinducer binding domain. We next queried the Hidden Markov Model (HMM) associated with autoinducer binding domains deposited in Pfam against the proteome of A. gephyra using HMMSEARCH ${ }^{47,48}$. The most significant hit (E-value 0.0015) a PAS domain S-box-containing protein also annotated as a GAF-domain-containing protein (WP_053066299.1) does not include significant sequence homology with LuxR-type, AHL receptors.

Utilizing blastp, the genome of A. gephyra was also assessed for features homologous to the alternative AHL receptors AinR (AAW85531.1) and LuxN (BAF43687.1) ${ }^{49,50}$, which do not include the conserved autoinducer binding domain associated with LuxR. No homologues with significant homology ( $>30 \%$ identity) for either alternative receptor were observed. The only resulting features were an annotated response regulator (WP_047859337.1; 43\% coverage and 27\% identity with AinR) and an annotated MASE1 domain-containing protein (WP_047860847.1; 60\% coverage and 20.04\% identity with LuxN). Although the annotated MASE1 domain-containing protein is predicted to include an $\mathrm{N}$-terminal transmembrane region, considered to be the recognition site for AHL quorum signals in LuxN, the only homology was associated with the C-terminal response regulatory receiver domain (PF00072.24) of LuxN.

Similar analysis of Vitiosangium sp. GDMCC 1.1324 provided a highly homologous LuxR-type receptor (WP_108076247.1). While the AHL receptor identified in the genome of Vitiosangium sp. is not clustered near $v i t I$ as is typical of LuxI-LuxR type synthase-receptor pairs, we cannot assume both are unpaired orphans and instead consider VitI might not be a truly solo AHL synthase (Supplemental Figure 4). Interestingly, the solo LuxR from Escherichia coli SdiA (PRK10188) ${ }^{51}$ was the highest scoring domain hit provided by blastp analysis of the LuxR from Vitiosangium sp. From these data we determined AgpI to be an orphaned AHL synthase without any cognate LuxR, AinR, or LuxN receptor present in the genome of A. gephyra, and despite the unclustered nature of the LuxR homologue identified in the genome of Vitiosangium sp., VitI and the annotated autoinducer synthase (WP_158502406.1) cannot be considered solo LuxI synthases without further investigation.

A. gephyra does not produce AHLs during axenic cultivation. Cultivation of A. gephyra on VY/2 agar plates at $30^{\circ} \mathrm{C}$ for 21 days provided fully developed, wispy myxobacterial swarms encompassing the entirety of the plate surface. Homogenized agar and cellular contents were extracted using traditional organic phase techniques to provide extracts for LC-MS/MS analysis. The resulting datasets from LC-MS/MS analysis of $A$. gephyra extracts were analyzed against datasets generated from analytical standards for a variety of AHLs including C9-AHL, C8-AHL, and C11-AHL to determine the presence of any produced AHL-like metabolites. Data from resulting mass spectra were scrutinized using the Global Natural Products Social Molecular Networking (GNPS) platform to generate molecular networks depicting similarities in detected metabolite scaffolds inferred from ionized fragment commonalities ${ }^{52}$. No metabolites that included the diagnostic AHL fragments at $102.0547 \mathrm{~m} / \mathrm{z}$ and $74.0599 \mathrm{~m} / \mathrm{z}$ associated with the core homoserine lactone moiety were detected in extracts from A. gephyra ${ }^{53,54}$. This data supports any one of the following conclusions A. gephyra does not produce AHLlike metabolites when grown axenically but may be active under other growth conditions; metabolites produced by AgpI do not possess structural similarity with typical AHL metabolites; or AgpI is simply nonfunctional. Silent or dormant BGCs are commonly observed during natural product discovery efforts, and various strategies to activate silent BGCs have been developed including addition of exogenous chemical elicitors and heterologous expression of silent BGCs in an alternative host $\mathrm{t}^{55,56}$.

Exogenous AHLs do not activate Agpl. Considering the typical autoinduction of LuxI synthases, we sought to determine if exogenous AHL metabolites might induce AgpI and provide observable AHL-like metabolites from A. gephyra. These experiments were conducted despite the absence of a feature that includes the conserved autoinducer domain encoded in the genome of A. gephyra in an effort to determine if a regulatory element with a non-typical AHL binding domain might induce expression of AgpI. Experiments introducing the deuterated AHLs $N$-hexanoyl-L-homoserine lactone- $d 3$ (C6-AHL- $d 3$ ) and $N$-butyryl-L-homoserine lactone- $d 5$ (C4-AHL-d5) to A. gephyra plates at $30 \mu \mathrm{M}$ after two weeks of growth at $30^{\circ} \mathrm{C}$ were conducted to determine if exogenous AHLs induce AgpI activity. Deuterium-labelled analogs of C6-AHL and C4-AHL were utilized to provide the ability to decouple exogenous signals from structurally similar AHLs potentially induced by exogenous AHL introduction. Using LC-MS/MS and molecular networking as previously described, no metabolites possessing the core homoserine lactone moiety were detected in the deuterated AHL-exposed extracts from $A$. gephyra suggesting that AgpI activity is not induced by exogenous AHLs. Ultimately, these experiments confirm that the exogenous AHL signals C6-AHL and C4-AHL do not induce biosynthesis of AHL metabolites from A. gephyra.

Heterologous expression of Agpl confirms functional production of AHLs. To explore the functionality of both AgpI and VitI and assumed biosynthesis of AHL-like metabolites, IPTG-inducible codon-optimized constructs of agpI and vitI included in replicating pET-28b $(+)$ plasmids suitable for expression in Escherichia coli BL21 were purchased. Heterologous expression of AgpI and VitI, subsequent extraction, LC-MS/MS analysis, and evaluation of molecular networks rendered by GNPS as previously described, provided a cluster family including 2 of 3 total nodes identified as C8-AHL $(228.159 \mathrm{~m} / \mathrm{z})$ and C9-AHL $(242.174 \mathrm{~m} / \mathrm{z})$ from internal GNPS public datasets as well as a third AHL metabolite detected at $226.144 \mathrm{~m} / \mathrm{z}$ (Fig. 3$)^{52}$. This cluster family was identical in both heterologous expression experiments suggesting that AgpI and VitI produce the same 3 AHL metabolites when heterologously expressed in E. coli with similar detected intensities for each AHL. Both C8-AHL and C9-AHL were confirmed to be present in AgpI and VitI extracts using analytical standards. Based on associated intensities, C8-AHL was the most abundant and the metabolite detected at $226.144 \mathrm{~m} / \mathrm{z}$ was the least abundant AHL. No AHL-like entities were detected in control extracts from E. coli containing no plasmid 


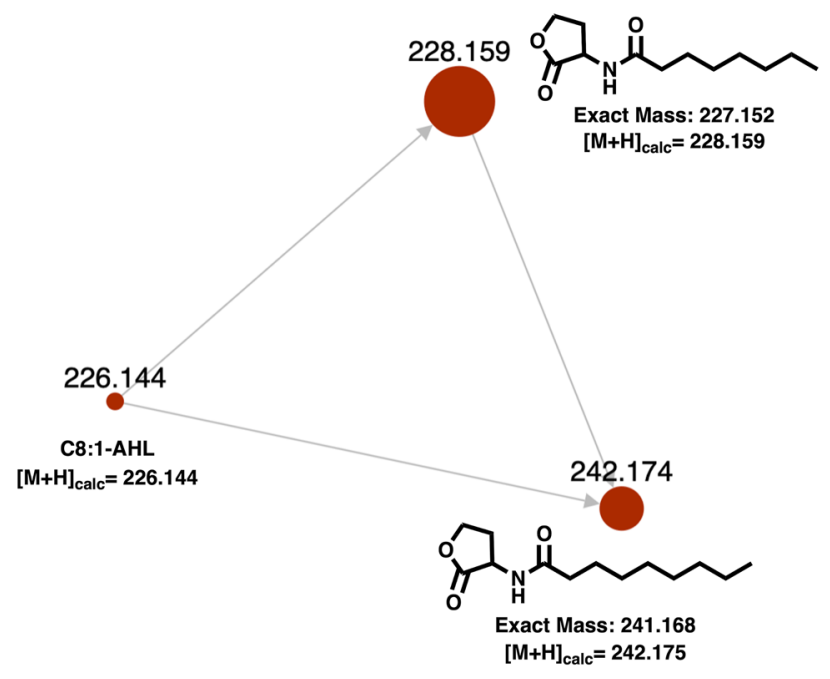

Figure 3. Molecular family from the molecular network of LC-MS/MS datasets from extracts of heterologous E. coli expressing AgpI rendered by GNPS (50). Detected m/z values from raw data positioned over each node with node diameter depicting associated intensities for each AHL.

and E. coli containing an empty pET28b expression plasmid (Supplemental Figures 5 and 6). From the mass difference between C8-AHL and the unknown AHL detected at $226.144 \mathrm{~m} / \mathrm{z}$ (2.015 Da measured vs. 2.01565 theoretical), as well as shared fragmentation patterns, we suggest the metabolite detected at $226.144 \mathrm{~m} / \mathrm{z}$ to likely be an unsaturated analog of C8-AHL (Fig. 4). From these experiments we determined that both AgpI and VitI are functional AHL synthases capable of producing the previously characterized AHLs C8-AHL and C9-AHL. These results suggest $A$. gephyra could produce AHLs and likely requires environmental cues or specific nutrients not present during our axenic cultivation conditions.

\section{Discussion}

Ultimately we conclude that the myxobacteria A. gephyra and Vitiosangium sp. encode functional AHL synthases that produce the AHL signals C8-AHL and C9-AHL when heterologously expressed in E. coli. Considering the strong precedent for heterologous expression of AHL synthases in E. coli to determine produced AHL metabolites, this suggests that both A. gephyra and Vitiosangium sp. are capable of producing one or all of the observed AHL signals and that AgpI is merely silent or cryptic during axenic cultivation of A. gephyra ${ }^{55,57-61}$. However, provided the subtle differences in LuxI synthase homologies and resulting chemical diversity of produced AHLs ${ }^{34}$, we should also consider that these synthases could instead utilize an acyl-ACP or CoA thioester precursor not available to the heterologous $E$. coli host, and we are actively exploring cultivation conditions that might induce native AHL production from A. gephyra ${ }^{55,59}$. While numerous bacteria have been observed to possess orphaned LuxR-type AHL receptors, a functional solo LuxI synthase without any cognate LuxR receptor also present in the genome has yet to be reported ${ }^{21,23,24,62}$. Although a functional orphaned LuxI-type synthase capable of producing AHLs has been reported from the sponge symbiont Ruegeria sp. KLH11, the strain also harbors 2 pairs of clustered LuxI/LuxR homologues ${ }^{23,63}$. We suggest that production of AHL quorum signals by myxobacteria would support the theoretical benefits of interspecies cross talk similar to functional, solo LuxR receptors ${ }^{24,64-66}$. We also propose that the more typical abundance of orphan LuxR receptors compared to the seemingly exceptional solo LuxI synthase reported here might correlate with the rarity of cooperative generalist predators ${ }^{21,28}$. The absence of any AHL metabolites during axenic cultivation of A. gephyra suggests an unknown regulatory mechanism independent from a LuxR receptor might be involved. However, previously reported eavesdropping by $M$. xanthus and response to exogenous AHLs despite the absence of any AHL receptor with homology to LuxR suggests myxobacteria may possess an undiscovered, alternative means of AHL detection and response ${ }^{18}$. An alternative explanation for the absence of AHL metabolites in the extracts of A. gephyra would be that AgpI is merely a non-functional feature that has been acquired but is not utilized. Although this seems unlikely due to the presence of additional LuxI-type synthases in the genome of Vitiosangium sp. and the understanding that specialized metabolite biosynthetic genes are often silent or unexpressed during axenic cultivation ${ }^{12,55,56}$, this explanation should be considered until either myxobacterium is observed to produce AHLs metabolites. While the benefit afforded predatory myxobacteria remains unclear, production of AHL signals known to regulate QS-associated physiological functions such as biofilm formation, specialized metabolism, and motility offers some insight ${ }^{15}$. Predatory disruption of any one of these functions would likely benefit the fitness of $A$. gephyra by improving predation of quorum signaling prey. For example, Pseudomonas putida biofilm formation is negatively regulated by the presence of AHLs ${ }^{67,68}$, and biofilm formation is commonly associated with predator avoidance ${ }^{69,70}$. Myxobacterial production of AHLs would therefore inhibit biofilm formation of $P$. putida which would benefit predation. However, without direct evidence of myxobacterial AHL production any interplay between AHL biosynthesis and predator-prey interactions remains hypothetical. Overall, we consider the presence of 2 functional 

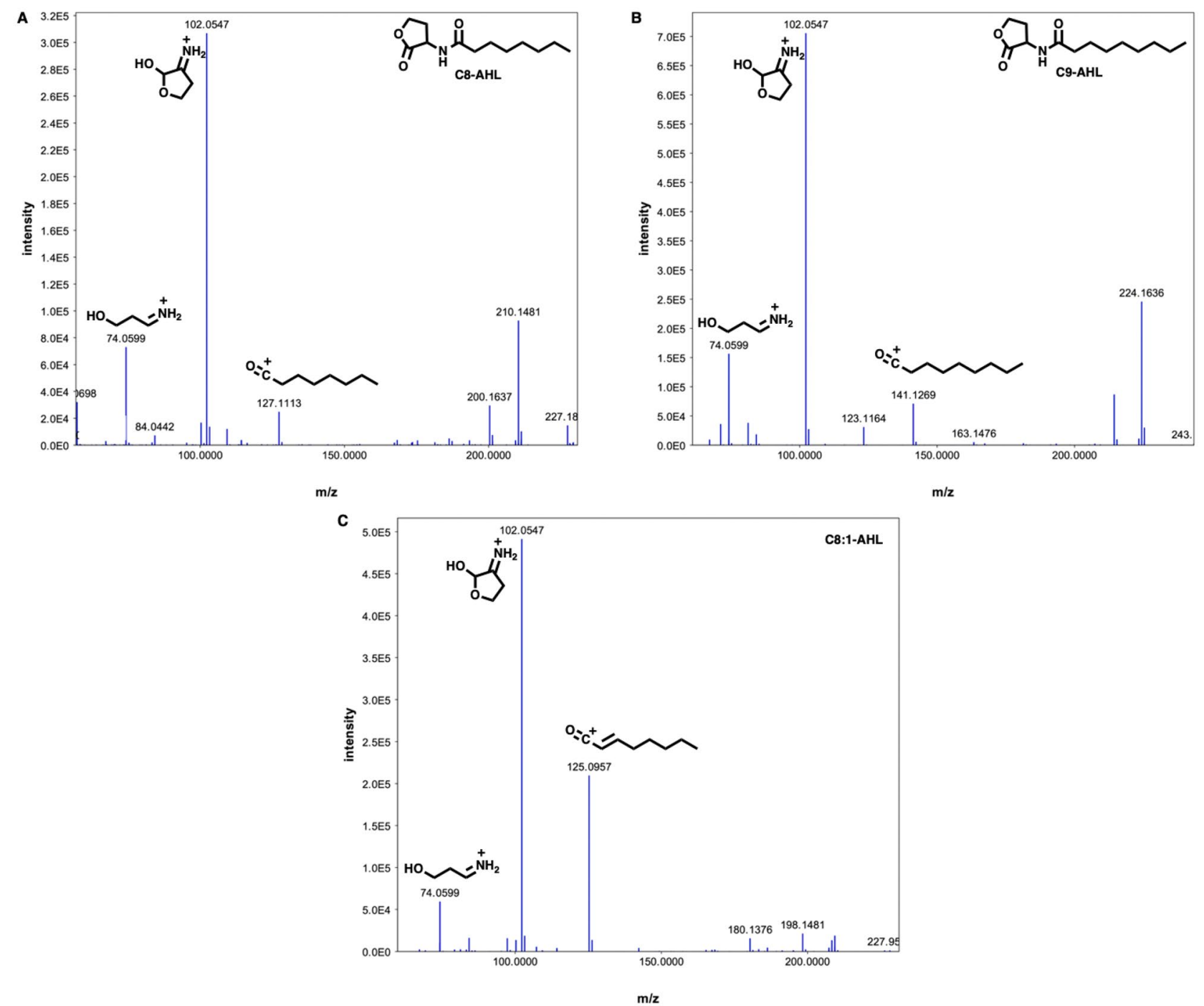

$\mathbf{m} / \mathbf{z}$

Figure 4. MS/MS fragmentation spectra with diagnostic fragments indicated for each AHL detected in extracts from heterologous E. coli expressing AgpI.

AHL synthases within the genomes of 2 predatory myxobacteria provides a unique perspective and supports the continued investigation of small molecule interactions that contribute to microbial community structures.

\section{Materials and methods}

Cultivation of A. gephyra. Archangium gephyra (DSM 2261) was initially obtained from German Collection of Microorganisms in Braunschweig was grown at $30^{\circ} \mathrm{C}$ on VY/2 agar $(5 \mathrm{~g} / \mathrm{L}$ baker's yeast, $1.36 \mathrm{~g} / \mathrm{L} \mathrm{CaCl}$, $0.5 \mathrm{mg} / \mathrm{L}$ vitamin $\mathrm{B}_{12}, 15 \mathrm{~g} / \mathrm{L}$ agar, $\mathrm{pH} 7.2$ ).

Bioinformatic assessment of Agpl and Vitl. The amino acid sequence for AgpI (WP_047862734.1) was submitted for blastp analysis against the non-redundant protein sequences database. The amino acid sequences for AgpI (WP_047862734.1) and VitI (WP_108069305.1) were submitted to EFI-EST analysis (https://efi.igb.illin ois.edu/efi-est/) to construct a sequence similarity network (SSN) of LuxI-type AHL synthases (PF00765) using the default settings. Results from EFI-EST analysis were visualized using Cytoscape (3.8.2) and are provided as supplemental data. Genomic contexts of AgpI (WP_047862734.1), VitI (WP_108069305.1), and the most homologous GNAT family N-acetyltransferases/autoinducer synthesis proteins according to blastp: including (WP_158502406.1) from Vitiosangium sp., (WP_119746752.1) from Paracoccus sp., (WP_055459978.1) from Chelatococcus sambhunathii, and from Mesorhizobium alhagi were analyzed to calculate GC\% of each acetyltransferases/autoinducer synthase and the surrounding genes (Fig. 1 and Supplemental Figure 2). Alignments from ClustalW, minimum evolution, and maximum likelihood phylogenetic trees were rendered using either MEGA 7 or MEGA X ${ }^{71-74}$. BiG-SCAPE (https://git.wageningenur.nl/medema-group/BiG-SCAPE) was was utilized locally to generate a sequence similarity network of all BGCs containing putative LuxI AHL synthases downloaded from the antiSMASH database (v2). The resulting network (Supplemental Figure 3) included a total 6627 nodes and 195,239 edges and was generated using the default parameters in BiG-SCAPE. 
Autoinducer binding site search. All 3014 domains annotated as autoinducer binding domains (PF03472) deposited in Pfam were subjected to blastp analysis against the A. gephyra genome (NZ_CP011509.1). For HMMSEARCH analysis, the raw HHM for autoinducer binding domains was downloaded from Pfam (PF03472) and utilized as input for profile-HMM vs protein sequence database via HMMSEARCH with the taxonomy restrictions set to limit analysis to A. gephyra or Vitiosangium sp. The amino acid sequence for AinR (AAW85531.1) and LuxN (BAF43687.1) was submitted for blastp analysis against the A. gephyra (NZ_CP011509) and Vitiosangium sp. (NZ_PZOX00000000.1) genomes.

Heterologous expression of Agpl and Vitl in E. coli. Constructs of AgpI and VitI codon optimized for expression in E. coli situated in pET28b were purchased from Genscript (Piscataway, NJ). Sequence data for these constructs are provided as supplemental data. The heterologous host E. coli K207-3 was transformed with each plasmid individually by electroporation (BTX Gemini Sc2, Harvard apparatus) to provide an E. coli strain capable of expressing AgpI and an E. coli strain capable of expressing VitI. In addition, an E. coli negative control was generated by transforming E. coli K207-3 with an empty pET28b vector (no AHL synthase construct). The transformed $E$. coli strains were grown at $37^{\circ} \mathrm{C}$ in LB broth supplemented with $50 \mu \mathrm{g} / \mathrm{mL}$ kanamycin, induced with IPTG (final concentration of $1.0 \mathrm{mM}$ ) at $\mathrm{OD}_{600}=0.6$, and grown overnight at $14{ }^{\circ} \mathrm{C}$ with shaking to facilitate heterologous protein expression. E. coli K207-3 with no included plasmid was also included as a negative control and was grown without the addition of kanamycin but otherwise under the same conditions.

Metabolite extraction and analysis. After 21 days of cultivation, A. gephyra plates were manually diced and extracted with excess EtOAc. Pooled EtOAc was filtered and dried in vacuo to provide crude extracts for LC-MS/MS analysis. Extracts from controls and heterologous strains of E. coli were generated by Amberlite XAD-16 absorber resin (Alfa Aesar) facilitated extraction of clarified culture broths following cell lysis. Resins were removed by filtration and were eluted with $\mathrm{MeOH}$ to provide extracts for LC-MS/MS analysis. Extraction for all strains were performed in triplicate.

LC-MS/MS analysis of the extracted samples was performed on an Orbitrap Fusion instrument (Thermo Scientific, San Jose, CA) controlled with Xcalibur version 2.0.7 and coupled to a Dionex Ultimate 3000 nanoUHPLC system. Samples were loaded onto a PepMap $100 \mathrm{C} 18$ column $(0.3 \mathrm{~mm} \times 150 \mathrm{~mm}, 2 \mu \mathrm{m}$, Thermo Fisher Scientific). Separation of the samples was performed using mobile phase A $(0.1 \%$ formic acid in water $)$ and mobile phase $B(0.1 \%$ formic acid in acetonitrile) at a rate of $6 \mu \mathrm{L} / \mathrm{min}$. The samples were eluted with a gradient consisting of 5 to $60 \%$ solvent B over $15 \mathrm{~min}$, ramped to $95 \%$ B over $2 \mathrm{~min}$, held for $3 \mathrm{~min}$, and then returned to $5 \% \mathrm{~B}$ over $3 \mathrm{~min}$ and held for $8 \mathrm{~min}$. All data were acquired in positive ion mode. Collision-induced dissociation (CID) was used to fragment molecules, with an isolation width of $3 \mathrm{~m} / \mathrm{z}$ units. The spray voltage was set to $3600 \mathrm{~V}$, and the temperature of the heated capillary was set to $300^{\circ} \mathrm{C}$. In CID mode, full MS scans were acquired from $\mathrm{m} / \mathrm{z} 150$ to 1200 followed by eight subsequent MS2 scans on the top eight most abundant peaks. The orbitrap resolution for both the MS1 and MS2 scans was 120,000. The expected mass accuracy was $<3 \mathrm{ppm}$. All extracts from heterologous expression experiments for AgpI and VitI performed in triplicate were analyzed with AHL production confirmed in each as described. Extracted-ion chromatographs depicting C8- and C9-AHL detection from E. coli expressing AgpI and E. coli expressing VitI and absence of detectible quantities of either AHL in extracts from negative controls included as Supplemental Figures 5 and 6.

Exogenous AHL exposure experiments. Stock solutions (10 mM) of $N$-hexanoyl-L-homoserine lactone- $d 3$ (C6-AHL- $d 3$ ) and $N$-butyryl-L-homoserine lactone- $d 5$ (C4-AHL- $d 5$ ) (Cayman Chemical) were prepared in DMSO. The required volumes of these stock solutions were filter sterilized and added to 14 days growing A. gephyra plates to give a final concentration of $30 \mu \mathrm{M}$. After 7 days of exogenous AHL exposure, A. gephyra plates were manually diced, extracted with excess EtOAc, and submitted to LC-MS/MS analysis as previously described.

GNPS dataset. Generated data were converted to .mzXML files using MS-Convert and mass spectrometry molecular networks were generated using the GNPS platform (http://gnps.ucsd.edu) $)^{52}$. LC-MS/MS data for this analysis were also deposited in the MassIVE Public GNPS data set (MSV000084574).

Supplementary material. Sequence data for codon-optimized AgpI and VitI constructs and Supplemental Figures 1-6 are included as supplementary material.

Received: 11 November 2020; Accepted: 20 January 2021

Published online: 04 February 2021

\section{References}

1. Cao, P., Dey, A., Vassallo, C. N. \& Wall, D. How myxobacteria cooperate. J Mol Biol 427, 3709-3721. https://doi.org/10.1016/j. jmb.2015.07.022 (2015).

2. Mohr, K. I. Diversity of myxobacteria-we only see the tip of the iceberg. Microorganisms 6, 84. https://doi.org/10.3390/microorgan isms6030084 (2018).

3. Munoz-Dorado, J., Marcos-Torres, F. J., Garcia-Bravo, E., Moraleda-Munoz, A. \& Perez, J. Myxobacteria: moving, killing, feeding, and surviving together. Front Microbiol 7, 781. https://doi.org/10.3389/fmicb.2016.00781 (2016). 
4. Bader, C. D., Panter, F. \& Muller, R. In depth natural product discovery - Myxobacterial strains that provided multiple secondary metabolites. Biotechnol Adv 39, 107480. https://doi.org/10.1016/j.biotechadv.2019.107480 (2019).

5. Baltz, R. H. Gifted microbes for genome mining and natural product discovery. J Ind Microbiol Biotechnol 44, 573-588. https:// doi.org/10.1007/s10295-016-1815-x (2017).

6. Herrmann, J., Fayad, A. A. \& Muller, R. Natural products from myxobacteria: novel metabolites and bioactivities. Nat Prod Rep 34, 135-160. https://doi.org/10.1039/c6np00106h (2017).

7. Korp, J., Vela Gurovic, M. S. \& Nett, M. Antibiotics from predatory bacteria. Beilstein J Org Chem 12, 594-607. https://doi. org/10.3762/bjoc.12.58 (2016).

8. Landwehr, W., Wolf, C. \& Wink, J. Actinobacteria and myxobacteria-two of the most important bacterial resources for novel antibiotics. Curr Top Microbiol Immunol 398, 273-302. https://doi.org/10.1007/82_2016_503 (2016).

9. Blin, K., Medema, M. H., Kottmann, R., Lee, S. Y. \& Weber, T. The antiSMASH database, a comprehensive database of microbial secondary metabolite biosynthetic gene clusters. Nucleic Acids Res 45, D555-D559. https://doi.org/10.1093/nar/gkw960 (2017).

10. Blin, K. et al. The antiSMASH database version 2: a comprehensive resource on secondary metabolite biosynthetic gene clusters. Nucleic Acids Res 47, D625-D630. https://doi.org/10.1093/nar/gky1060 (2019).

11. Blin, K. et al. antiSMASH 5.0: updates to the secondary metabolite genome mining pipeline. Nucleic Acids Res 47, W81-W87. https://doi.org/10.1093/nar/gkz310 (2019).

12. Gregory, K., Salvador, L. A., Akbar, S., Adaikpoh, B. I. \& Stevens, D. C. Survey of biosynthetic gene clusters from sequenced myxobacteria reveals unexplored biosynthetic potential. Microorganisms 7, 181. https://doi.org/10.3390/microorganisms706018 1 (2019).

13. Lang, E., Schumann, P., Tindall, B. J., Mohr, K. I. \& Sproer, C. Reclassification of Angiococcus disciformis, Cystobacter minus and Cystobacter violaceus as Archangium disciforme comb. nov., Archangium minus comb. nov. and Archangium violaceum comb. nov., unification of the families Archangiaceae and Cystobacteraceae, and emended descriptions of the families Myxococcaceae and Archangiaceae. Int J Syst Evol Microbiol 65, 4032-4042. https://doi.org/10.1099/ijsem.0.000533 (2015).

14. Sharma, G. \& Subramanian, S. Unravelling the complete genome of Archangium gephyra DSM 2261T and evolutionary insights into myxobacterial chitinases. Genome Biol Evol 9, 1304-1311. https://doi.org/10.1093/gbe/evx066 (2017).

15. Papenfort, K. \& Bassler, B. L. Quorum sensing signal-response systems in gram-negative bacteria. Nat Rev Microbiol 14, 576-588. https://doi.org/10.1038/nrmicro.2016.89 (2016).

16. Brotherton, C. A., Medema, M. H. \& Greenberg, E. P. LuxR homolog-linked biosynthetic gene clusters in proteobacteria. mSystems 3, e00208. https://doi.org/10.1128/mSystems.00208-17 (2018).

17. Whitworth, D. E. \& Zwarycz, A. A genomic survey of signalling in the myxococccaceae. Microorganisms 8, 1739. https://doi. org/10.3390/microorganisms8111739 (2020).

18. Lloyd, D. G. \& Whitworth, D. E. The myxobacterium Myxococcus xanthus can sense and respond to the quorum signals secreted by potential prey organisms. Front Microbiol 8, 439. https://doi.org/10.3389/fmicb.2017.00439 (2017).

19. Hudaiberdiev, S. et al. Census of solo LuxR genes in prokaryotic genomes. Front Cell Infect Microbiol 5, 20. https://doi.org/10.3389/ fcimb.2015.00020 (2015).

20. Schuster, M., Sexton, D. J., Diggle, S. P. \& Greenberg, E. P. Acyl-homoserine lactone quorum sensing: from evolution to application. Annu Rev Microbiol 67, 43-63. https://doi.org/10.1146/annurev-micro-092412-155635 (2013).

21. Subramoni, S. \& Venturi, V. LuxR-family "solos": bachelor sensors/regulators of signalling molecules. Microbiology 155, 1377-1385. https://doi.org/10.1099/mic.0.026849-0 (2009).

22. Subramoni, S. \& Venturi, V. PpoR is a conserved unpaired LuxR solo of Pseudomonas putida which binds N-acyl homoserine lactones. BMC Microbiol 9, 125. https://doi.org/10.1186/1471-2180-9-125 (2009).

23. Tobias, N. J. et al. New vocabulary for bacterial communication. ChemBioChem https://doi.org/10.1002/cbic.201900580 (2019).

24. Wellington, S. \& Greenberg, E. P. Quorum sensing signal selectivity and the potential for interspecies cross talk. MBio 10, e00146. https://doi.org/10.1128/mBio.00146-19 (2019).

25. Whiteley, M., Diggle, S. P. \& Greenberg, E. P. Progress in and promise of bacterial quorum sensing research. Nature 551, 313-320. https://doi.org/10.1038/nature24624 (2017).

26. Mendes-Soares, H. \& Velicer, G. J. Decomposing predation: testing for parameters that correlate with predatory performance by a social bacterium. Microb Ecol 65, 415-423. https://doi.org/10.1007/s00248-012-0135-6 (2013).

27. Morgan, A. D., MacLean, R. C., Hillesland, K. L. \& Velicer, G. J. Comparative analysis of myxococcus predation on soil bacteria. Appl Environ Microbiol 76, 6920-6927. https://doi.org/10.1128/AEM.00414-10 (2010).

28. Perez, J., Moraleda-Munoz, A., Marcos-Torres, F. J. \& Munoz-Dorado, J. Bacterial predation: 75 years and counting!. Environ Microbiol 18, 766-779. https://doi.org/10.1111/1462-2920.13171 (2016).

29. Awal, R. P. et al. Vitiosangium cumulatum gen. nov., sp. nov. and Vitiosangium subalbum sp. nov., soil myxobacteria, and emended descriptions of the genera Archangium and Angiococcus, and of the family Cystobacteraceae. Int J Syst Evol Microbiol 67, 14221430. https://doi.org/10.1099/ijsem.0.001829 (2017).

30. Badhai, J., Whitman, W. B. \& Das, S. K. Draft genome sequence of Chelatococcus sambhunathii strain HT4T (DSM 18167T) isolated from a hot spring in India. Genome Announc 4, e00825. https://doi.org/10.1128/genomeA.00825-16 (2016).

31. Panday, D. \& Das, S. K. Chelatococcus sambhunathii sp. nov., a moderately thermophilic alphaproteobacterium isolated from hot spring sediment. Int J Syst Evol Microbiol 60, 861-865. https://doi.org/10.1099/ijs.0.013466-0 (2010).

32. Brader, G., Sjoblom, S., Hyytiainen, H., Sims-Huopaniemi, K. \& Palva, E. T. Altering substrate chain length specificity of an acylhomoserine lactone synthase in bacterial communication. J Biol Chem 280, 10403-10409. https://doi.org/10.1074/jbc.M4086 03200 (2005).

33. Chakrabarti, S. \& Sowdhamini, R. Functional sites and evolutionary connections of acylhomoserine lactone synthases. Protein Eng 16, 271-278. https://doi.org/10.1093/proeng/gzg031 (2003).

34. Dong, S. H. et al. Molecular basis for the substrate specificity of quorum signal synthases. Proc Natl Acad Sci U S A 114, 9092-9097. https://doi.org/10.1073/pnas.1705400114 (2017).

35. Eberl, L. et al. Involvement of N-acyl-L-hormoserine lactone autoinducers in controlling the multicellular behaviour of Serratia liquefaciens. Mol Microbiol 20, 127-136. https://doi.org/10.1111/j.1365-2958.1996.tb02495.x (1996).

36. Milton, D. L. et al. Quorum sensing in Vibrio anguillarum: characterization of the vanI/vanR locus and identification of the autoinducer N-(3-oxodecanoyl)-L-homoserine lactone. J Bacteriol 179, 3004-3012. https://doi.org/10.1128/jb.179.9.3004-3012.1997 (1997).

37. Nhu Lam, M. et al. Insights into beta-ketoacyl-chain recognition for beta-ketoacyl-ACP utilizing AHL synthases. Chem Commun (Camb) 54, 8838-8841. https://doi.org/10.1039/c8cc04532a (2018).

38. Parsek, M. R., Schaefer, A. L. \& Greenberg, E. P. Analysis of random and site-directed mutations in rhII, a Pseudomonas aeruginosa gene encoding an acylhomoserine lactone synthase. Mol Microbiol 26, 301-310. https://doi.org/10.1046/j.1365-2958.1997.57419 35.x (1997).

39. Pearson, J. P. et al. Structure of the autoinducer required for expression of Pseudomonas aeruginosa virulence genes. Proc Natl Acad Sci U S A 91, 197-201. https://doi.org/10.1073/pnas.91.1.197 (1994).

40. Rasch, M. et al. Involvement of bacterial quorum-sensing signals in spoilage of bean sprouts. Appl Environ Microbiol 71, 3321-3330. https://doi.org/10.1128/AEM.71.6.3321-3330.2005 (2005). 
41. Schaefer, A. L. et al. A new class of homoserine lactone quorum-sensing signals. Nature 454, 595-599. https://doi.org/10.1038/ nature07088 (2008).

42. White, C. E. \& Winans, S. C. Cell-cell communication in the plant pathogen Agrobacterium tumefaciens. Philos Trans R Soc Lond B Biol Sci 362, 1135-1148. https://doi.org/10.1098/rstb.2007.2040 (2007).

43. Engebrecht, J. \& Silverman, M. Identification of genes and gene products necessary for bacterial bioluminescence. Proc Natl Acad Sci U S A 81, 4154-4158. https://doi.org/10.1073/pnas.81.13.4154 (1984).

44. Zallot, R., Oberg, N. \& Gerlt, J. A. The EFI web resource for genomic enzymology tools: leveraging protein, genome, and metagenome databases to discover novel enzymes and metabolic pathways. Biochemistry 58, 4169-4182. https://doi.org/10.1021/acs.bioch em.9b00735 (2019).

45. Navarro-Munoz, J. C. et al. A computational framework to explore large-scale biosynthetic diversity. Nat Chem Biol 16, 60-68. https://doi.org/10.1038/s41589-019-0400-9 (2020).

46. El-Gebali, S. et al. The Pfam protein families database in 2019. Nucleic Acids Res 47, D427-D432. https://doi.org/10.1093/nar/ gky995 (2019).

47. Finn, R. D., Clements, J. \& Eddy, S. R. HMMER web server: interactive sequence similarity searching. Nucleic Acids Res 39, W2937. https://doi.org/10.1093/nar/gkr367 (2011).

48. Wheeler, T. J. \& Eddy, S. R. nhmmer: DNA homology search with profile HMMs. Bioinformatics 29, 2487-2489. https://doi. org/10.1093/bioinformatics/btt403 (2013).

49. Gilson, L., Kuo, A. \& Dunlap, P. V. AinS and a new family of autoinducer synthesis proteins. J Bacteriol 177, 6946-6951. https:// doi.org/10.1128/jb.177.23.6946-6951.1995 (1995).

50. Lupp, C., Urbanowski, M., Greenberg, E. P. \& Ruby, E. G. The Vibrio fischeri quorum-sensing systems ain and lux sequentially induce luminescence gene expression and are important for persistence in the squid host. Mol Microbiol 50, 319-331. https://doi. org/10.1046/j.1365-2958.2003.t01-1-03585.x (2003).

51. Sitnikov, D. M., Schineller, J. B. \& Baldwin, T. O. Control of cell division in Escherichia coli: regulation of transcription of ftsQA involves both rpoS and SdiA-mediated autoinduction. Proc Natl Acad Sci U S A 93, 336-341. https://doi.org/10.1073/pnas.93.1.336 (1996).

52. Wang, M. et al. Sharing and community curation of mass spectrometry data with Global Natural Products Social Molecular Networking. Nat Biotechnol 34, 828-837. https://doi.org/10.1038/nbt.3597 (2016).

53. Gould, T. A., Herman, J., Krank, J., Murphy, R. C. \& Churchill, M. E. Specificity of acyl-homoserine lactone synthases examined by mass spectrometry. J Bacteriol 188, 773-783. https://doi.org/10.1128/JB.188.2.773-783.2006 (2006).

54. Patel, N. M., Moore, J. D., Blackwell, H. E. \& Amador-Noguez, D. Identification of unanticipated and novel N-Acyl L-Homoserine lactones (AHLs) using a sensitive non-targeted LC-MS/MS method. PLoS ONE 11, e0163469. https://doi.org/10.1371/journ al.pone.0163469 (2016).

55. Mao, D., Okada, B. K., Wu, Y., Xu, F. \& Seyedsayamdost, M. R. Recent advances in activating silent biosynthetic gene clusters in bacteria. Curr Opin Microbiol 45, 156-163. https://doi.org/10.1016/j.mib.2018.05.001 (2018).

56. Xu, F., Nazari, B., Moon, K., Bushin, L. B. \& Seyedsayamdost, M. R. Discovery of a cryptic antifungal compound from Streptomyces albus J1074 using high-throughput elicitor screens. J Am Chem Soc 139, 9203-9212. https://doi.org/10.1021/jacs.7b02716 (2017).

57. Britstein, M. et al. A new N-Acyl homoserine lactone synthase in an uncultured symbiont of the red sea sponge Theonella swinhoei. Appl Environ Microbiol 82, 1274-1285. https://doi.org/10.1128/AEM.03111-15 (2016).

58. Dang, H. T., Komatsu, S., Masuda, H. \& Enomoto, K. Characterization of LuxI and LuxR protein homologs of N-Acylhomoserine lactone-dependent quorum sensing system in Pseudoalteromonas sp. 520P1. Mar Biotechnol (NY) 19, 1-10. https://doi.org/10.1007/ s10126-016-9726-4 (2017).

59. Hoshino, S., Onaka, H. \& Abe, I. Activation of silent biosynthetic pathways and discovery of novel secondary metabolites in actinomycetes by co-culture with mycolic acid-containing bacteria. J Ind Microbiol Biotechnol 46, 363-374. https://doi.org/10.1007/ s10295-018-2100-y (2019).

60. Neumann, A., Patzelt, D., Wagner-Dobler, I. \& Schulz, S. Identification of new N-acylhomoserine lactone signalling compounds of Dinoroseobacter shibae DFL-12(T) by overexpression of luxI genes. ChemBioChem 14, 2355-2361. https://doi.org/10.1002/ cbic.201300424 (2013).

61. Zheng, H. et al. A LuxR/LuxI-type quorum-sensing system in a plant bacterium, Mesorhizobium tianshanense, controls symbiotic nodulation. J Bacteriol 188, 1943-1949. https://doi.org/10.1128/JB.188.5.1943-1949.2006 (2006).

62. Chugani, S. \& Greenberg, E. P. An evolving perspective on the Pseudomonas aeruginosa orphan quorum sensing regulator QscR. Front Cell Infect Microbiol 4, 152. https://doi.org/10.3389/fcimb.2014.00152 (2014).

63. Zan, J. et al. A solo luxI-type gene directs acylhomoserine lactone synthesis and contributes to motility control in the marine sponge symbiont Ruegeria sp. KLH11. Microbiology 161, 50-56. https://doi.org/10.1099/mic.0.083956-0 (2015).

64. McDougald, D., Srinivasan, S., Rice, S. A. \& Kjelleberg, S. Signal-mediated cross-talk regulates stress adaptation in Vibrio species. Microbiology 149, 1923-1933. https://doi.org/10.1099/mic.0.26321-0 (2003).

65. Noel, J. T. et al. Salmonella SdiA recognizes N-acyl homoserine lactone signals from Pectobacterium carotovorum in vitro, but not in a bacterial soft rot. Mol Plant Microbe Interact 23, 273-282. https://doi.org/10.1094/MPMI-23-3-0273 (2010).

66. Venturi, V., Bertani, I., Kerenyi, A., Netotea, S. \& Pongor, S. Co-swarming and local collapse: quorum sensing conveys resilience to bacterial communities by localizing cheater mutants in Pseudomonas aeruginosa. PLoS ONE 5, e9998. https://doi.org/10.1371/ journal.pone.0009998 (2010).

67. Arevalo-Ferro, C., Reil, G., Gorg, A., Eberl, L. \& Riedel, K. Biofilm formation of Pseudomonas putida IsoF: the role of quorum sensing as assessed by proteomics. Syst Appl Microbiol 28, 87-114. https://doi.org/10.1016/j.syapm.2004.10.005 (2005).

68. Fazli, M. et al. Regulation of biofilm formation in Pseudomonas and Burkholderia species. Environ Microbiol 16, 1961-1981. https ://doi.org/10.1111/1462-2920.12448 (2014).

69. Seiler, C. et al. Grazing resistance of bacterial biofilms: a matter of predators' feeding trait. FEMS Microbiol Ecol 93, fix112. https ://doi.org/10.1093/femsec/fix112 (2017).

70. Sun, S., Noorian, P. \& McDougald, D. Dual role of mechanisms involved in resistance to predation by protozoa and virulence to humans. Front Microbiol 9, 1017. https://doi.org/10.3389/fmicb.2018.01017 (2018).

71. Kumar, S., Stecher, G. \& Tamura, K. MEGA7: molecular evolutionary genetics analysis version 7.0 for bigger datasets. Mol Biol Evol 33, 1870-1874. https://doi.org/10.1093/molbev/msw054 (2016).

72. Sievers, F. \& Higgins, D. G. Clustal omega for making accurate alignments of many protein sequences. Protein Sci 27, 135-145. https://doi.org/10.1002/pro.3290 (2018).

73. Kumar, S., Stecher, G., Li, M., Knyaz, C. \& Tamura, K. MEGA X: molecular evolutionary genetics analysis across computing platforms. Mol Biol Evol 35, 1547-1549. https://doi.org/10.1093/molbev/msy096 (2018).

74. Stecher, G., Tamura, K. \& Kumar, S. Molecular evolutionary genetics analysis (MEGA) for macOS. Mol Biol Evol 37, 1237-1239. https://doi.org/10.1093/molbev/msz312 (2020).

\section{Acknowledgements}

The authors appreciate funding and support from the National Institute of Allergy and Infectious Diseases (R15AI137996), Fulbright (H.A), and the University of Mississippi. 


\section{Author contributions}

H.A., M.D., and S.K.M. conducted the experiments; H.A. and D.C.S. wrote the manuscript; H.A., M.D., S.K.M., J.S.S., and D.C.S. edited the manuscript. All authors have read and agreed to the published version of the manuscript.

\section{Competing interests}

The authors declare no competing interests.

\section{Additional information}

Supplementary Information The online version contains supplementary material available at https://doi. org/10.1038/s41598-021-82480-1.

Correspondence and requests for materials should be addressed to D.C.S.

Reprints and permissions information is available at www.nature.com/reprints.

Publisher's note Springer Nature remains neutral with regard to jurisdictional claims in published maps and institutional affiliations.

(c) (i) Open Access This article is licensed under a Creative Commons Attribution 4.0 International License, which permits use, sharing, adaptation, distribution and reproduction in any medium or format, as long as you give appropriate credit to the original author(s) and the source, provide a link to the Creative Commons licence, and indicate if changes were made. The images or other third party material in this article are included in the article's Creative Commons licence, unless indicated otherwise in a credit line to the material. If material is not included in the article's Creative Commons licence and your intended use is not permitted by statutory regulation or exceeds the permitted use, you will need to obtain permission directly from the copyright holder. To view a copy of this licence, visit http://creativecommons.org/licenses/by/4.0/.

(C) The Author(s) 2021 